\title{
A GOLDEN RATIO AS A GENERALIZATION OF THE 2/3 RULE OF JANUSZ LYKO
}

\author{
Arkadiusz Maciuk, Antoni Smoluk
}

\begin{abstract}
A golden ratio has been applied since ancient times in many areas, starting with architecture and art, and nowadays in modern financial markets and contemporary physics. This paper defines a golden rule in a set of preferences, generalizing the problem of group choice and the $2 / 3$ rule of Łyko by means of a golden number corresponding to the ratio of lengths of sides in a golden rectangle.
\end{abstract}

Keywords: golden number, problem of group choice, 2/3 rule.

JEL Classification: C02

DOI: $10.15611 / \mathrm{me} .2018 .14 .03$

\section{Number $\chi$}

Mathematics and any science are aimed at getting to know the world, perfecting human kind, and achieving formal perfection. Isomorphism is an essential term in mathematics, preserving the structure and giving objects a new content. What is a rectangle? This paper defines a rectangle as an ordered pair of strongly positive numbers. If the first number is greater than the second one, then such a rectangle is said to be horizontal, and if the second number is greater than the first one, the rectangle is said to be vertical. Certainly, a rectangle with sides of equal lengths is a square. One can cut off the squares with sides equal to a shorter side of the rectangle. Such cutting can go on as long as a vertical rectangle becomes horizontal or, the other way around, a horizontal rectangle becomes vertical. A rectangle is called a golden rectangle if the ratio of its shorter side to its longer side is a golden number:

$$
\chi=\frac{\sqrt{5}-1}{2} \text {. }
$$

Arkadiusz Maciuk

Wrocław University of Economics e-mail: arkadiusz.maciuk@ue.wroc.pl ORCID: 0000-0001-5208-1074

\author{
Antoni Smoluk \\ Wrocław University of Economics \\ e-mail: math@ue.wroc.pl \\ ORCID: 0000-0002-9990-037X
}


The golden number $\chi$ represents roughly 61.8 percent of a unity, i.e. less than $\frac{2}{3}$. Let $(b, a)$ denote a golden rectangle whose shorter side is $a$, hence $a=\chi b$. Rectangles are all points from the first quadrant of the plane $\mathbb{R}^{2}$ excluding the border, i.e. the points $(x, y)$, where $x, y \in \mathbb{R}_{+}^{*}$. The latter symbol denotes a multiplicative group of strongly positive real numbers. The elements of this group are numbers, and each of them represents a class of equivalent rectangles - as it is a ratio of the sides of the rectangle. The numbers smaller than 1 represent a class of horizontal rectangles, those greater than 1 - a class of vertical rectangles, and the unity - a class of squares. Golden rectangles have the property that one can remove just one square section at each step to pass from a horizontal rectangle to a vertical one, or vice versa. Let $x_{0}=b, y_{0}=a, x_{n+1}=y_{n}, y_{n+1}=x_{n}-y_{n}$ where $n \in \mathbb{N}$. A recursive sequence of subsequently removed squares defines a linear operator $A$ with matrix $\left(\begin{array}{cc}0 & 1 \\ 1 & -1\end{array}\right)$; it yields therefore $\left(\begin{array}{c}x_{n} \\ x_{n+1}\end{array}\right)=A\left(\begin{array}{l}x_{n} \\ y_{n}\end{array}\right)$. The eigenvalues of the matrix $A$ are $\chi$ and $-\chi^{-1}$.

A golden rectangle, or a golden ratio, has been present in art and science for 2,400 years. The flags of most countries, and a sheet of paper, have the shape of a golden rectangle. Such a ratio of sides in a rectangle can often be found in nature, both on the macro scale of galaxies as well as on the micro scale. For the human eye the shape is esthetic and natural. For centuries it has been fascinating and inspiring scores of outstanding people, and today science keeps on developing its abundant applications [see: Omotehinwa, Ramon 2013] in such diverse areas as neurobiology [Weiss, Weiss 2003; Carracedo et al. 2008], physics [Coldea et al. 2010; Sigalotti, Mejias 2006], and the financial markets [Boroden 2008]. The ratio of sides in a golden rectangle was denoted in ancient Greece by the letter $\chi$, where $\chi=\frac{\sqrt{5}-1}{2}$, and such notation is adopted in this paper. This notation is different from the one currently used in the English-language literature following the important contribution of Mark Barr who introduced the first letter of the Greek sculptor Phidias's name $\varphi$ as the symbol for the golden ratio, where $\varphi=\frac{1+\sqrt{5}}{2}$, and $\chi$ itself is written as $\frac{1}{\varphi}=\Phi$. 


\section{A continued fraction and the Euclidean sequence}

A process of removing squares from a rectangle defines a continued fraction. If at the first step $n_{0}$ squares are removed from the rectangle, $n_{1}$ squares at the second step, and so on, then a sequence $\left\{n_{k}\right\}$ of natural numbers is obtained. If the process of removing squares does not terminate, we obtain a proper continued fraction, otherwise we obtain a rational number, a finite fraction. When does the process of removals terminate? It does when the last removed section is also a square. The remaining numbers in the sequence $\left\{n_{k}\right\}$ are then zeroes. Each sequence of such natural numbers defines a continued fraction. A continued fraction is a sequence of rational functions $\left\{f_{k}\right\}$ of the form $f_{k}(x)=\frac{1}{n_{k}+x}$, when $n_{k}$ is not equal to zero; whereas if $n_{k+1}$ is zero, then $f_{k}$ is identically zero. A continued fraction is typically identified with the sequence of numbers $\left(f_{0}(0), f_{0}\left(f_{1}(0)\right), f_{0}\left(f_{1}\left(f_{2}(0)\right)\right), \ldots\right)$. A continued fraction is symbolically written as an expression of the form $\frac{1}{n_{0}+\frac{1}{n_{1}+\frac{1}{1+\cdots}}}$.

The value of a continued fraction is the limit of a given sequence of real numbers, if it exists. A continued fraction obtained from a golden rectangle is made of a sequence of numbers 1 , because at each step only one square is removed from this rectangle. The limit of its corresponding continued fraction is a golden number. Since this fraction is of the form $\frac{1}{1+\frac{1}{1+\frac{1}{1+\cdots}}}$, therefore if $x$ denotes the value of this fraction, then we get the equality $1=\frac{1}{1+x}$. The solution to this equation is given by the eigenvalues of operator $A$, hence one of the solutions is a golden number. Each square corresponds with the sequence $(1,0,0, \ldots)$, hence the value of this fraction is always 1 , regardless of the lengths of the sides of the squares. The two rectangles $(b, a)$ and $\left(b_{1}, a_{1}\right)$ are equivalent with respect to the operation of removing the squares, if $(b, a)=$ $p\left(b_{1}, a_{1}\right)$, i.e. when the sides of those rectangles are proportional. Each pair of equivalent rectangles determines the same continued fraction. The limit of such a fraction is the ratio of the shorter side to the longer side. When the process of removing the squares is terminated, then the sides of the rectangle are commensurable. Such a rectangle can be divided into a finite number of squares. If a rectangle cannot be divided into a finite number of squares, then the ratio of its sides is an irrational number. 
Definition. Euclid's sequence is a sequence of natural numbers all of whose terms are nonzero or a sequence whose first term is a natural nonzero number, and if any term is zero, then each following term of this sequence is also zero.

The sequences of natural numbers generated by cutting rectangles are certainly Euclid's sequences. An essential question concerns the existence of Euclid's sequences which cannot be obtained by removing squares from a rectangle.

A continued fraction can also be seen in a more general way; it is a pair of two Euclid's sequences, where the first one can be finite and with zero terms, and the second one has all nonzero terms. If $a=\left(a_{n}\right)=\left(a_{0}, a_{1}, \ldots\right)$ is the first sequence, and $b=\left(b_{n}\right)$ the second one, then a continued fraction defined by these sequences is of the form:

$$
\frac{a_{0}}{b_{0}+\frac{a_{1}}{b_{1}+\frac{a_{2}}{b_{2}+\cdots}} \text {. }}
$$

If $g_{k}(x)=\frac{a_{k}}{b_{k}+x}$, this continued fraction can be written as follows: $\left(g_{0}(0), g_{0}\left(g_{1}(0)\right), g_{0}\left(g_{1}\left(g_{2}(0)\right)\right), \ldots\right)$. The terms of this sequence are called convergent of a continued fraction. Let $r_{k}$ denote the $k$-th convergent. It is a rational number of the form $\frac{p_{k}}{q_{k}}$. We provide recursive formulas for a numerator and a denominator of the fraction $r_{k}=\frac{p_{k}}{q_{k}}$, where $k \in \mathbb{N}$. Let

$$
p_{0}=a_{0}, q_{0}=b_{0}, p_{1}=a_{0} b_{1}, q_{1}=a_{1}+b_{0} b_{1} ;
$$

then

$$
p_{k+2}=b_{k+2} p_{k+1}+a_{k+2} p_{k}, q_{k+2}=b_{k+2} q_{k+1}+a_{k+2} q_{k} .
$$

The proof of these equalities is by induction. We verify them for $k=0$. We have

$$
r_{2}=\frac{p_{2}}{q_{2}}=\frac{a_{0}}{b_{0}+\frac{a_{1}}{b_{1}+\frac{a_{2}}{b_{2}}}}=\frac{a_{0} b_{1} b_{2}+a_{0} a_{2}}{a_{2} b_{0}+b_{2}\left(a_{1}+b_{0} b_{1}\right)}=\frac{b_{2} p_{1}+a_{2} p_{0}}{b_{2} q_{1}+a_{2} q_{0}} .
$$

Likewise, we have $p_{2}=b_{2} p_{1}+a_{2} p_{0}$ and $q_{2}=b_{2} q_{1}+a_{2} q_{0}$. After simple transforming, we demonstrate the truth. Let us now assume the truth for $k+2$, to demonstrate the truth for $k+3$. By definition we have

$$
r_{k+3}=\frac{\left(b_{k+2}+\frac{a_{k+3}}{b_{k+3}}\right) p_{k+1}+a_{k+2} p_{k}}{\left(b_{k+2}+\frac{a_{k+3}}{b_{k+3}}\right) q_{k+1}+a_{k+2} q_{k}} .
$$


After rewriting we have

$$
\begin{gathered}
\frac{\left(b_{k+2} b_{k+3}+a_{k+3}\right) p_{k+1}+b_{k+3} a_{k+2} p_{k}}{\left(b_{k+2} b_{k+3}+a_{k+3}\right) q_{k+1}+b_{k+3} a_{k+2} q_{k}}=\frac{b_{k+3}\left(b_{k+2} p_{k+1}+a_{k+2} p_{k}\right)+a_{(k+3)} p_{k+1}}{b_{k+3}\left(b_{k+2} q_{k+1}+a_{k+2} q_{k}\right)+a_{(k+3)} q_{k+1}}, \text { i.e. } \\
r_{k+3}=\frac{b_{k+3} p_{k+2}+a_{k+3} p_{k+1}}{b_{k+3} q_{k+2}+a_{k+3} q_{k+1}}
\end{gathered}
$$

and this proves the truth of formulas for $p_{k}$ and $q_{k}$. Given the golden number, the terms of both sequences $a$ and $b$ are all number 1 . It yields therefore $r_{0}=1$, $r_{1}=\frac{1}{2}, r_{2}=\frac{2}{3}, r_{3}=\frac{3}{5}$, and generally, $r_{k}=\frac{\phi_{k+1}}{\phi_{k+2}}$, where $\phi_{k}$ denotes a Fibonacci sequence defined by induction: $\phi_{0}=0, \phi_{1}=1, \phi_{k+2}=\phi_{k}+\phi_{k+1}$, where $k \in \mathbb{N}$. Thus, the formula $\lim \frac{\phi_{k}}{\phi_{k+1}}=\chi$ results directly.

\section{The $2 / 3$ rule and a golden rule}

A preference is a reflexive and transitive relation, while a preference that is also weakly asymmetric represents an order. The $2 / 3$ rule proposed by $€$ yko [2000] and Łyko, Misztal, Smoluk [2000] specifies when a maximal relation established from a finite set of preferences is a preference. All preferences relate to the same set of elements that can be called commodities. Given a finite population and its members with their own preferences, how to create a reasonable group preference? One of the methods to solve this task is to create a maximal preference. There is a maximal relation between commodity $x$ and commodity $y$ when the majority of population prefer $y$ to $x$. A maximal relation is not always a preference. The $2 / 3$ rule provides a sufficient condition for this relation to be a preference. The pair $(x, y)$ is called a decision. If each decision in a maximal relation is taken with a frequency strongly greater than $\frac{2}{3}$, then the maximal relation is a preference.

Łyko's proof contains a slight inaccuracy. The statement implies such preferences that are orders, hence it is not generally true as claimed in the article. This paper aims at providing a more general rule, called a golden rule, with number $\chi$ playing an essential role. We go on assuming that all preferences are orders. What condition must be satisfied by a maximal relation to be a group order? This relation is certainly reflexive and weakly asymmetric, but it can be intransitive. Transitivity implies that if most people prefer $y$ to $x$, and $z$ to $y$, then they should also prefer $z$ to $x$. For this to be really the case, the frequencies of decisions $(x, y)$ and $(y, z)$ must be greater than the golden number $\chi$, and furthermore, the probability of the product of both decisions 
should equal the product of individual probabilities, i.e. these decisions should be independent events. If they are, then $P(x, y)>\chi, P(y, z)>\chi$ and $P((x, y),(y, z))=P(x, y) P(y, z)>\chi^{2}$, but $\chi^{2}=1-\chi$. Thus, the probability of the decision 'I prefer $x$ to $z$ ' is smaller than $1-\chi$. Therefore, decision $(x, z)$ must be an element of the maximal relation. The golden rule means that when the frequencies of maximal decisions are greater than the golden number, and when individual decisions regarded as events are independent, the maximal relation is an order. The golden rule extends the $2 / 3$ rule of $Ł y k o$. We also fix an error regarding preferences, since the $2 / 3$ rule is valid for orders only, not for preferences.

\section{Bibliography}

Boroden C. (2008). Fibonacci Trading: How to Master the Time and Price Advantage. McGraw-Hill. New York.

Carracedo L.M., Kaiser M., Kramer M.A. et al. (2008). Temporal interactions between cortical rhythms. Frontiers in Neuroscience 2(2), pp. 145-154.

Coldea R., Tennant D.A., Wheeler E.M. et al. (2010). Quantum Criticality in an Ising Chain: Experimental Evidence for Emergent $E_{8}$ Symmetry. Science 327(5962), pp. 177-180.

Łyko J. (2000). Twierdzenia Arrowa a ordynacje. [In:] Smoluk A. (ed.). Elementy metrologii ekonomicznej. Wydawnictwo AE we Wrocławiu, pp. 165-168.

Łyko J., Misztal A., Smoluk A. (2000). Problem of group choice and the 2/3 rule. Ekonomia Matematyczna 4. Wydawnictwo AE we Wrocławiu, pp. 21-34.

Omotehinwa T.O., Ramon S.O. (2013). Fibonacci numbers and golden ratio in mathematics and science. International Journal of Computer and Information Technology 2(4), pp. 630-638.

Sigalotti L.D.G., Mejias A. (2006). The golden ratio in special relativity. Chaos, Solitons and Fractals 30(3), pp. 521-524.

Weiss V., Weiss H. (2003). The golden mean as clock cycle of brain waves. Chaos, Solitons and Fractals 18(4), pp. 643-652. 